\title{
Multiorgan Involvement in COVID-19 and Possible Therapies
}

\author{
Prachee Sathe $^{1} \quad$ Vijay Sundar Singh ${ }^{2}$ \\ ${ }^{1}$ Department of Critical Care Medicine, Ruby Hall Clinic, \\ Grant Medical Foundation, Pune, Maharashtra, India \\ ${ }^{2}$ Department of Critical Care Medicine, DY Patil Medical College, \\ Pune, Maharashtra, India \\ J Card Crit Care:2020;4:20-24
}

\begin{abstract}
Address for correspondence Prachee Sathe, MD FRCP, Director ICU Ruby Hall Clinic, Pune, Maharashtra, 411001, India

(e-mail: prachee.sathe@gmail.com).
\end{abstract}

\begin{abstract}
In late 2019, China reported cases of respiratory illness in humans, which involved a novel Coronavirus SARS-CoV-2 (also known as 2019-nCoV). The World Health

Keywords

- COVID-19

- multiorgan

involvement

- possible therapies Organization (WHO) termed the disease COVID-19 (i.e., Coronavirus disease 2019). Most of the morbidity and mortality from COVID-19 is largely due to acute viral pneumonitis that leads to acute respiratory distress syndrome (ARDS). This article will discuss the clinical features of the multiorgan involvement in COVID-19 as well as the management of patients who become critically ill due to COVID-19.
\end{abstract}

\section{Introduction}

In late 2019, China reported cases of respiratory illness in humans, which involved a novel Coronavirus SARS-CoV-2 (also known as 2019-nCoV). The World Health Organization (WHO) termed the disease COVID-19 (i.e., Coronavirus disease 2019). Most of the morbidity and mortality from COVID-19 is largely due to acute viral pneumonitis that leads to acute respiratory distress syndrome (ARDS). This article will discuss the clinical features of the multiorgan involvement in COVID-19 as well as the management of patients who become critically ill due to COVID- 19 .

\section{Causative Agent and Epidemiology}

COVID-19 is caused by a $\beta$ coronavirus named SARS-CoV- 2 . Corona viruses are enveloped viruses with a single positive-stranded RNA genome ( $26-32 \mathrm{~kb}$ in length). The structure of the receptor-binding gene region is very similar to that of the SARS coronavirus, and the virus has been shown to use the same receptor, the angiotensin-converting enzyme 2 (ACE2), for cell entry. ${ }^{2}$ The disease has a mean incubation period of 5 days, median of 3 to 4 days, with wide range up to 24 days (common range 2-7 days). The number of people diagnosed with COVID-19 worldwide crossed the 3 million mark on April 27, 2020; and 3.4\% was the mortality rate estimate by the WHO as of March $3 .{ }^{3} \mathrm{~A}$ review by the WHO-China Joint Mission of 55, 924 laboratory-confirmed cases in China said $6.1 \%$ were classified as critical (respiratory failure, shock, and multiple organ dysfunction or failure) and $13.8 \%$ as severe (dyspnea, respiratory rate $\geq 30$ breaths per min, oxygen saturation $\leq 93 \%$, partial pressure of arterial oxygen to fraction of inspired oxygen [PaO2/FiO2] ratio 50\% within 24-48 hours). ${ }^{4}$ In Italy, until March 29, 2020, up to 12 to $14 \%$ of all positive cases required ICU admission. ${ }^{5,6}$

\section{Clinical Features}

The median time from symptom onset to ICU transfer is around 12 days. Timing of onset of sepsis from onset of illness is approximately 9 days (range 7-13 days), while ARDS typically presents at around 12 days (range $8-15$ days) from the onset of illness. ${ }^{7}$ SARS-CoV-2 is known to cause multiorgan dysfunction, so it is important that the clinician is aware of the organs it affects and the way it presents.

\section{ARDS}

Autopsy studies of COVID-19 associated lung disease have demonstrated bilateral diffuse alveolar damage with cellular fibromyxoid exudates, desquamation of pneumocytes, pulmonary edema, and hyaline membrane formation. These studies show that there is also some evidence of direct viral injury to lung tissue, not just due to inflammatory sequelae. ${ }^{8}$ Some patients with COVID-related lung disease have significantly higher compliance than that is typical for their shunt published online

13 July 2020
DOI https://doi.org/

10.1055/s-0040-1714151 ISSN 2457-0206.
(C)2020 Official Publication of The Simulation Society (TSS), accredited by International Society of Cardiovascular Ultrasound (ISCU).
License terms

() (1) $\odot \circledast$ 
fraction, which indicates this may be a very different phenotype than typical ARDS. The explanation remains unclear, with dysregulation of pulmonary perfusion considered a possible explanation, as postulated by Gattinoni et al. ${ }^{9}$

According to Gattinoni et al, there are two types of COVID-19 patients with ARDS. In type-1 patients, severe hypoxemia is always associated with a respiratory system compliance of $>50 \mathrm{~mL} / \mathrm{cmH}_{2} \mathrm{O}$. The lung's gas volume is high and the recruitability is minimal. In 20 to $30 \%$ of the type 2 patients admitted to the intensive care unit (ICU), severe hypoxemia is associated with compliance values $<40 \mathrm{~mL} / \mathrm{cmH}_{2} \mathrm{O}$, indicating severe ARDS. ${ }^{10}$

\section{Cardiac Involvement}

Various forms of cardiac involvement such as acute cardiac injury, arrhythmias, pericarditis, myocarditis and, possibly, acute coronary syndrome (ACS) are known to occur in COVID19 patients. The definition of acute cardiac injury differs in studies and is nonspecific. More recent studies define as troponin > 99th percentile upper limit of normal; earlier studies include abnormal ECG or echocardiographic findings.,11 The mechanism through which SARS-CoV-2 causes cardiac injury is unknown, although several have been proposed, based on very limited data outside of case series and reports.

A) Viral invasion into cardiac myocytes causing possible direct toxicity (i.e., myocarditis).

B) ACS and demand ischemia.

C) Stress cardiomyopathy (i.e., Takotsubo's).

D) Profound inflammatory response/cytokine storm, leading to viral invasion into cardiac myocytes ${ }^{12-14}$

The occurrence of unspecified arrhythmias in $17 \%$ of hospitalized patients with COVID-19 ( $n=23$ of 138) have been reported in case series. Higher rates were observed in ICU patients $(44 \%, n=16)$ compared with non-ICU patients ( $7 \%$, $n=7) .{ }^{15}$ The rate of ventricular arrhythmia (VT)/ventricular fibrillation (VF) was $5.9 \%$ in a study of 189 hospitalized patients in Wuhan, China. ${ }^{16}$

There is nil current available data on the incidence of ACS in COVID. However, it is presumed that due to the presence of ACE2 receptors on the endothelium, and the known increased risk of ACS in influenza, there is a possible increased incidence of ACS among COVID-19 patients.

\section{Neurological Involvement}

There is much still to be learned about the central nervous system (CNS) involvement of COVID-19, but lessons from scientific and clinical experience from other human Coronaviruses suggest neuroinvasive potential of SARS$\mathrm{CoV}-2$.The incidence of neurologic manifestations varies between 36.4 to $69 \%$ of hospitalized COVID-19 patients. Delirium, confusion, or executive dysfunction are particularly common, and may occur in a majority of patients. Other common neurologic manifestations were dizziness (16.8\%), headache (13.1\%), impaired consciousness (7.5\%), hypogeusia (5.6\%), hyposmia $(5.1 \%)$, or stroke $(2.8-5 \%) .{ }^{17.18}$ New case reports of rarer complications are emerging that have reported Guillain-Barre Syndrome (GBS), ${ }^{19}$ Miller-Fisher Syndrome (MFS), ${ }^{20}$ encephalitis, ${ }^{21}$ and acute necrotizing encephalopathy. ${ }^{22}$

\section{Liver Disease}

Present literature reveals that approximately 15 to $50 \%$ of patients with COVID-19 have abnormal liver function tests (LFTs). ${ }^{23,24}$ The variability in the incidence may be explained by the differences in disease severity, multiple etiologies of hepatic injury (such as direct viral injury, indirect inflammatory injury, and drug-induced injury), as well as nonhepatic causes of elevated transaminases. The pattern of injury is predominately hepatocellular. Elevations are of almost all liver enzymes are seen with AST, ALT and LDH elevations being the most common. Liver injury associated with COVID19 is often mild, even in patients with severe disease, and is self- resolving. ${ }^{25}$ To date, acute liver failure has not been reported, even in the severely ill and those with chronic liver disease. . $^{2426}$

\section{Acute Kidney Injury (AKI)}

The incidence of AKI in COVID-19 varies widely, but estimates range from $0.5 \%$ to $27 \% .25,27$ The wide range of estimates of AKI incidence may reflect different populations included in studies (e.g., ward vs. ICU patients). The most likely etiology of AKI in COVID-19 is acute tubular necrosis (ATN). An autopsy series from China found severe ATN and interstitial infiltration with $\mathrm{CD} 68+$ macrophages on histopathologic examination of kidney tissue. Membrane attack complex protein (C5b-9) deposition was also seen in the tubules, suggesting that activation of the alternative complement pathway may play a role in tubular injury as well. ACE2 receptors in renal vasculature may also play a role for specific nephrotoxicity.

\section{Hematological Involvement}

In ICU patients, cumulative incidences range from $20 \%$ to $40 \%$ in patients on varying levels of prophylactic anticoagulation. ${ }^{28}$ Higher D-dimer and fibrin and fibrinogen degradation product (FDP) levels track with multiorgan dysfunction syndrome and poorer prognosis. ${ }^{7,15}$ The mechanism for venous thromboembolism (VTE) is not known currently and likely due to multifactorial reasons such as systemic inflammatory response syndrome (SIRS), as seen in sepsis, and possible direct endothelial damage from viral injury/ACE2 binding. Disseminated intravascular coagulation (DIC) was found in 16 of 183 hospitalized patients in Wuhan. Median time to onset of DIC was 4 days into hospital admission. ${ }^{29}$

\section{Cytokine Activation Syndrome}

Apart from septic shock and cardiogenic shock, cytokine activation syndrome per se can also present as circulatory shock in COVID-19 patients. A subgroup of patients with severe COVID-19 may have cytokine activation syndrome 
and secondary hemophagocytic lymphohistiocytosis (HLH). ${ }^{30}$ Patients who had cytokine activation developed rapid progression to ARDS, shock, and multiorgan failure. Neutrophil activation likely contributes to the pathogenesis of cytokine storm with elevated IL6 levels and ARDS. Wu et al found that COVID-19 confirmed patients with ARDS have higher neutrophil counts, average 7.04 (95\% CI: 3.98 to 10.12) versus those without ARDS, whose average is 3.06 (2.03 to 5.56). ${ }^{31}$ Similar patterns of cytokine storm and ARDS have been seen with SARS and MERS. Other studies have suggested that increased proinflammatory cytokines in the serum are associated with pulmonary injury in SARS, MERS, and COVID-19.

\section{Diagnosis}

There is multifold rise in the number of areas with community transmission worldwide and also substantial risk of missing cases early in a local outbreak; therefore, ICU practitioners should have a very high-index of suspicion and a low-threshold for diagnostic testing for any patient presenting with severe acute respiratory infection. Diagnosis is based on RT-PCR assays for severe acute respiratory syndrome coronavirus 2 (SARS-CoV-2). Falsely negative upper respiratory tract samples are seen in patients with pneumonia. ${ }^{32}$ Sampling from the lower respiratory tract is recommended by WHO, such as with sputum and endotracheal aspirates. These procedures potentially generate aerosol and must be done with strict airborne precautions. Bronchoscopy should generally be avoided if possible to minimize exposure of healthcare workers to SARS-CoV-2, although the diagnostic yield of bronchoalveolar lavage for COVID-19 might be high. ${ }^{33}$ The sensitivity of RT-PCR assays for the critically ill patients is currently unknown. Repeated sampling might be required when initial tests are negative despite suspicious clinical features.

\section{Management ARDS}

\section{Before Intubation}

The management options available are supplemental oxygen, continuous positive airway pressure (CPAP), noninvasive ventilation (NIV), high-flow nasal cannula (HFNC), and

Table 1 Ventilation strategies for different phenotypes in COVID-19

\begin{tabular}{|l|l|}
\hline $\begin{array}{l}\text { Type } 1 \text { (Minimal } \\
\text { recruitability) }\end{array}$ & Type 2 (Low compliance) \\
\hline $\begin{array}{l}\text { Consider lower PEEP }(<10 \mathrm{~cm} \\
\mathrm{H} 2 \mathrm{O})\end{array}$ & Use higher PEEP $(<15 \mathrm{~cm} \mathrm{H2O})$ \\
\hline $\begin{array}{l}\text { Can use more liberal tidal } \\
\text { volume }(7-9 \mathrm{~mL} / \mathrm{kg}) \text { as needed }\end{array}$ & $\begin{array}{l}\text { Lower tidal volume }(5-7 \\
\mathrm{mL} / \mathrm{kg}) \text { to be used }\end{array}$ \\
\hline Consider prone positioning & Implement prone positioning \\
\hline
\end{tabular}

Abbreviation: PEEP, positive end-expiratory pressure. awake prone positioning. Target nonvigorous breathing to avoid self-inflicted lung injury in patient (-Table $\mathbf{1}$ ).

\section{During Ventilation}

The goals of mechanical ventilation are to minimize pulmonary stress, optimize oxygenation, and avoid ventilator-induced lung injury. Consider extracorporeal membrane oxygenation (ECMO) in refractory hypoxemia.

Avoid excessive positive fluid balance. Transition to spontaneous breathing to be done cautiously, and spontaneous trials to be done only at the very end of the weaning process. ${ }^{34}$

\section{Other Intensive Care Management}

A) Blood cultures to be sent; empiric antibiotics to be considered (secondary infections reported are high).

B) Measurement of lactate levels; cautious fluids for hypovolemia; check preload responsiveness; echocardiography; vasopressors or inotropes to be added if needed.

C) To avoid routine use of corticosteroids.

D) Unnecessary patient transfers to be avoided; use point-ofcare tests such as ultrasound wherever possible.

E) Renal replacement therapy if indicated.

F) Protocolized sedation practice.

G) Early enteral nutrition and adequate glycemic control to be achieved.

H) Early physical therapy.

I) Prevention of nosocomial infections.

J) Deep vein thrombosis prophylaxis.

K) Stress ulcer prophylaxis. ${ }^{35}$

\section{Therapeutics in COVID-19}

\section{Remedesvir}

Remdesivir is a nucleotide prodrug metabolized to an ana$\log$ of adenosine triphosphate, which inhibits viral RNAdependent RNA polymerase, causing premature termination of RNA transcription. This drug has shown in vitro activity against SARS-CoV-2. ${ }^{36} \mathrm{~A}$ case series of compassionate use remdesivir which analyzed 53 patients, in whom $68 \%$ had improvement in their oxygen-support class, $47 \%$ were discharged, and $13 \%$ passed away. But without a control group to compare, it is unclear if the use of remdesivir altered the natural progression of COVID-19 disease in these patients. ${ }^{37}$

An NIH press release has commented on the adaptive NIAID trial that had evaluated 1063 patients with COVID19. This study was a randomized, placebo-controlled trial at 68 sites. Full details of the trial are not yet available, but remdesivir was reported to result in a median time to recovery of 11 days versus 15 days in the placebo group (31\% reduction, $p<0.001)$ and a mortality rate of $8 \%$ in the remdesivir arm versus $11.6 \%$ in the placebo arm $(p=0.059)$. A full publication is expected shortly.

\section{Hydroxychloroquine and Chloroquine}

Hydroxychloroquine (HCQ) is an antimalarial 4-aminoquinoline shown to have in vitro (but not yet in vivo) activity against diverse RNA viruses. HCQ is thought to act against viruses through multiple mechanisms such as inhibition 
of viral entry, inhibition of viral release into the host cell, reduction of viral infectivity, and immune modulation. Even though some unpublished small studies showed promise, ${ }^{38}$ recent studies have shown no difference in outcomes and some recent studies show even harm. ${ }^{39}$

\section{Lopinavir and Ritonavir (LPV/r)}

Lopinavir and ritonavir are both protease inhibitors. Lopinavir may theoretically work against coronaviruses like SARS-CoV-2 by inhibiting 3-chymotrypsin-like protease. A recent randomized, controlled, open-label trial which assessed lopinavir-ritonavir $(n=99)$ versus standard of care $(n=100)$ in SARS-CoV-2 patients found that treatment with $\mathrm{LPV} / \mathrm{r}$ was not associated with a difference in time to clinical improvement or mortality. However, randomization did not occur until a median of 13 days after symptom onset, so the window for benefit may have already closed. ${ }^{40}$

\section{Convalescent Plasma (Immunotherapy)}

Convalescent plasma is taken from patients who have previously recovered from a COVID-19 viral infection and are now able to donate their immunoglobulin-containing blood. The presumed mechanism of action is that antibodies present in convalescent plasma may suppress viremia. Shen et a ${ }^{41}$ reported on five critically ill COVID-19 patients who received convalescent plasma, four of five who saw temperature normalization within 3 days and three of five who have been discharged (days 51,53, and 55), with the other two in stable condition at the time of the publication. The most recent case series reported out on 10 severe COVID-19 disease patients who received convalescent plasma and tolerated it well. ${ }^{42}$

\section{Tocilizumab}

IL-6 activates $T$ cells and macrophages, among other cell types. IL-6 inhibitors are approved for cytokine activation syndrome complications related to Chimeric Antigen Receptor T cell (CAR-T) therapy. ${ }^{43}$ Even though unpublished studies report success, large randomized controlled trials are needed. In severe cases of COVID-19 with suspicion of cytokine activation syndrome, one can consider use in conjunction with rheumatology and infectious diseases consultation.

\section{Glucocorticoids}

The Centre for Disease Control and Prevention (CDC) and the WHO recommend that glucocorticoids should not be routinely administered to patients with COVID-19, unless there is a separate evidence-based indication in that patient (e.g., asthma or chronic obstructive lung disease exacerbation, refractory septic shock, and adrenal insufficiency). However, their administration in ICU patients with COVID-19-related ARDS is controversial.

\section{Others}

Intravenous immunoglobulin and favipiravir (RNAdependent RNA polymerase inhibitor) to date do not have peer-reviewed, published safety data available for SARS$\mathrm{CoV}-2$. Hence, these modalities are not recommended unless part of a clinical trial.
Convective therapies for removal of inflammatory cytokines during cytokine storm is another novel therapy considered for COVID-19 patients with severe inflammation Monitoring inflammation and antibodies are important, especially in patients infected by virus with persistent fever or abnormal coagulopathy. Expeditious control of the cytokine storm in early phase might be beneficial to selective patients, and case reports and small series from China and European countries have been published. ${ }^{44}$

A recent review by Ronco et al mentions that in the absence of established drugs or vaccines for COVID-19, pathophysiological rationale may support the application of the extracorporeal therapies which might help patients who are critically ill with COVID-19 with situations such as shocklike syndrome, the need for vasopressors and capillary leak syndrome, and laboratory criteria such as the levels of IL-6 and other cytokines, as a rescue therapy. ${ }^{45}$

\section{Conclusion}

Although COVID-19 starts as a respiratory illness, it has the possibility of affecting various organs directly (direct organotropism) or indirectly (systemic inflammatory processes or sepsis like syndromes), presenting a multitude of problems. On the one hand, atypical presentations to specific specialties and nonrecognition/missing of primary disorders (COVID-19) may put many HCWs at risk of exposure, on the other hand, it may complicate the clinical course of the patient.

Indirect involvement of various organs represents a more severe subset of patients, and lack of specific therapies puts them at higher mortality prediction, making case for wellplanned trials for novel therapies.

Conflicts of Interest

None declared.

\section{References}

1 World Health Organization. Director-General's remarks at the media briefing on 2019-nCoV on 11 February 2020. Available at: https://www.who.int/dg/speeches/detail/ who-director-general-s-remarks-at-the-media-briefing-on2019-ncov-on-11-february-2020. Accessed June 16, 2020

2 Zhou P, Yang XL, Wang XG, et al. A pneumonia outbreak associated with a new coronavirus of probable bat origin. Nature 2020;579(7798):270-273

3 Worldometer. COVID-19 coronavirus pandemic. Available at: https://www.worldometers.info/coronavirus/. Accessed April 30,2020

4 WHO-China Joint Mission. Report of the WHO-China Joint Mission on Coronavirus Disease 2019 (COVID-19). Available at: https://www.who.int/docs/default-source/coronaviruse/ who-china-joint-mission-on-covid-19-final-report.pdf Accessed June 16, 2020

5 Grasselli G, Pesenti A, Cecconi M. Critical care utilization for the COVID-19 outbreak in Lombardy, Italy: early experience and forecast during an emergency response. JAMA 2020;323(16):1545-1546

6 Remuzzi A, Remuzzi G. COVID-19 and Italy: what next. ?Lancet 2020;395(10231):1225-1228 
7 Zhou F, Yu T, Du R, et al. Clinical course and risk factors for mortality of adult inpatients with COVID-19 in Wuhan, China: a retrospective cohort study. Lancet 2020;395(10229) : 1054-1062

8 Xu Z, Shi L, Wang Y, et al. Pathological findings of COVID-19 associated with acute respiratory distress syndrome. Lancet Respir Med 2020;8(4):420-422

9 Gattinoni L, Coppola S, Cressoni M, Busana M, Rossi S, Chiumello D. COVID-19 does not lead to a "typical" acute respiratory distress syndrome. Am J Respir Crit Care Med 2020;201(10):1299-1300

10 Gattinoni L, Chiumello D, Rossi S. COVID-19 pneumonia: ARDS or not? Crit Care 2020;24(1):154

11 Shi S, Qin M, Shen B, et al. Association of Cardiac Injury With Mortality in Hospitalized Patients With COVID-19 in Wuhan, China. JAMA Cardiol 2020;(e-pub ahead of print). doi: 10.1001/ jamacardio.2020.0950

$12 \mathrm{Hu} \mathrm{H}$, Ma F, Wei X, Fang Y. Coronavirus fulminant myocarditis saved with glucocorticoid and human immunoglobulin. Eur Heart J 2020;(e-pub ahead of print). doi: 10.1093/eurheartj/ ehaa 190

13 Inciardi RM, Lupi L, Zaccone G, et al. Cardiac involvement in a patient with Coronavirus disease 2019 (COVID-19) JAMA Cardiol 2020;(e-pub ahead of print). doi: 10.1001/ jamacardio.2020.1096

14 Ruan Q, Yang K, Wang W, Jiang L, Song J. Clinical predictors of mortality due to COVID-19 based on an analysis of data of 150 patients from Wuhan, China. Intensive Care Med 2020;46(5):846-848

15 Wang D, Hu B, Hu C, et al. Clinical characteristics of 138 hospitalized patients with 2019 novel Coronavirus-infected pneumonia in Wuhan, China. JAMA 2020;323(11):1061-1069

16 Guo T, Fan Y, Chen M, et al. Cardiovascular implications of fatal outcomes of patients with Coronavirus disease 2019 (COVID19) JAMA Cardiol 2020;(e-pub ahead of print). doi: 10.1001/ jamacardio.2020.1017

17 Mao L, Jin H, Wang M, et al. Neurologic manifestations of hospitalized patients with Coronavirus disease 2019 in Wuhan, China. JAMA Neurol 2020;(e-pub ahead of print). doi: 10.1001/ jamaneurol.2020.1127

18 Helms J, Kremer S, Merdji H, et al. Neurologic features in severe SARS-CoV-2 infection. N Engl J Med 2020;382(23):2268-2270

19 Toscano G, Palmerini F, Ravaglia S, et al. Guillain-Barré Syndrome associated with SARS-CoV-2. N Engl J Med 2020 (e-pub ahead of print). doi: 10.1056/NEJMc2009191

20 Gutiérrez-Ortiz C, Méndez A, Rodrigo-Rey S, et al. Miller Fisher Syndrome and polyneuritis cranialis in COVID19. Neurology 2020;(e-pub ahead of print). doi: 10.1212/ WNL.0000000000009619

21 Moriguchi T, Harii N, Goto J, et al. A first case of meningitis/ encephalitis associated with SARS-Coronavirus-2. Int J Infect Dis 2020;94:55-58

22 Poyiadji N, Shahin G, Noujaim D, Stone M, Patel S, Griffith B. COVID-19-associated acute hemorrhagic necrotizing encephalopathy: CT and MRI features. Radiology 2020;201187:201187

23 Zhang C, Shi L, Wang FS. Liver injury in COVID-19: management and challenges. Lancet Gastroenterol Hepatol 2020;5(5):428-430

$24 \mathrm{Xu} \mathrm{L}$, Liu J, Lu M, Yang D, Zheng X. Liver injury during highly pathogenic human coronavirus infections. Liver Int 2020;40(5):998-1004

25 Guan WJ, Ni ZY, Hu Y, et al; China Medical Treatment Expert Group for Covid-19. Clinical characteristics of Coronavirus disease 2019 in China. N Engl J Med 2020;382(18):1708-1720

26 Ong J, Young BE, Ong S. COVID-19 in gastroenterology: a clinical perspective. Gut 2020;69(6):1144-1145
27 Diao B, Wang C, Wang R, et al. Human kidney is a target for novel severe acute respiratory syndrome Coronavirus 2 (SARS-CoV-2) infection. medRxiv 2020 (e-pub ahead of print). doi:10.1101/2020.03.04.20031120

28 Klok FA, Kruip MJHA, van der Meer NJM, et al. Incidence of thrombotic complications in critically ill ICU patients with COVID-19. Thromb Res 2020;191:145-147

29 Tang N, Li D, Wang X, Sun Z. Abnormal coagulation parameters are associated with poor prognosis in patients with novel coronavirus pneumonia. J Thromb Haemost 2020;18(4):844-847

30 Mehta P, McAuley DF, Brown M, Sanchez E, Tattersall RS, Manson JJ; HLH Across Speciality Collaboration, UK. COVID19: consider cytokine storm syndromes and immunosuppression. Lancet 2020;395(10229): 1033-1034

31 Wu C, Chen X, Cai Y, et al. Risk factors associated with acute respiratory distress syndrome and death in patients with Coronavirus disease 2019 pneumonia in Wuhan, China. JAMA Intern Med 2020 (e-pub ahead of print).doi: 10.1001/ jamainternmed.2020.0994

32 Ai T, Yang Z, Hou H, et al. Correlation of chest CT and RT-PCR testing in Coronavirus disease 2019 (COVID-19) in China: a report of 1014 cases. Radiology 2020;200642:200642

33 Wang W, Xu Y, Gao R, et al. Detection of SARS-CoV-2 in Different Types of Clinical Specimens. JAMA 2020;323(18):1843-1844

34 Marini JJ, Gattinoni L. Management of COVID-19 respiratory distress. JAMA 2020;(e-pub ahead of print). doi: 10.1001/ jama.2020.6825

35 Phua J, Weng L, Ling L, et al; Asian Critical Care Clinical Trials Group. Intensive care management of coronavirus disease 2019 (COVID-19): challenges and recommendations. Lancet Respir Med 2020;8(5):506-517

36 Wang M, Cao R, Zhang L, et al. Remdesivir and chloroquine effectively inhibit the recently emerged novel coronavirus (2019-nCoV) in vitro. Cell Res 2020;30(3):269-271

37 Grein J, Ohmagari N, Shin D, et al. Compassionate use of remdesivir for patients with severe Covid-19. N Engl J Med 2020 (e-pub ahead of print. doi: 10.1056/NEJMoa2007016

38 Chen Z, Hu, J, Zhang, Z, et al Efficacy of hydroxychloroquine in patients with COVID-19: results of a randomized clinical trial. MedRxiv2020;(e-pub ahead of print. doi: https://doi.org/10.1 101/2020.03.22.20040758

39 Borba MGS, Val FF, Sampaio VS, et al. Effect of high vs low doses of chloroquine diphosphate as adjunctive therapy for patients hospitalized with severe acute respiratory syndrome Coronavirus 2 (SARS-CoV-2) infection: a randomized clinical trial. JAMA Netw Open 2020;3(4):e208857

40 Cao B, Wang Y, Wen D, et al. A trial of lopinavir-ritonavir in adults hospitalized with severe Covid-19. N Engl J Med 2020;382(19):1787-1799

41 Shen C, Wang Z, Zhao F, et al. Treatment of 5 critically ill patients with COVID-19 with convalescent plasma. JAMA 2020;323(16):1582-1589

42 Duan K, Liu B, Li C, et al. Effectiveness of convalescent plasma therapy in severe COVID-19 patients. Proc Natl Acad Sci U S A 2020;117(17):9490-9496

43 Brudno JN, Kochenderfer JN. Recent advances in CAR T-cell toxicity: Mechanisms, manifestations and management. Blood Rev 2019;34:45-55

44 Ma J, Xia P, Zhou Y, et al. Potential effect of blood purification therapy in reducing cytokine storm as a late complication of critically ill COVID-19. Clin Immunol 2020;214:108408

45 Ronco C, Reis T. Kidney involvement in COVID-19 and rationale for extracorporeal therapies. Nat Rev Nephrol 2020;16(6):308-310 